\title{
Creating Media with Prescribed Permeability Using the Asymptotic Solution to EM Wave Scattering Problem
}

\author{
Alexander Ramm \\ Kansas State University \\ Manhattan, KS, 66506-2602, USA \\ E-mail: ramm@math.ksu.edu
}

\begin{abstract}
Asymptotic solution to scattering problem of electromagnetic (EM) waves by many small impedance particles, embedded in a homogeneous medium, is applied for creating media with prescribed permeability. Physical properties of the particles are described by their boundary impedances. The limiting equation is obtained for the effective EM field in the resulting medium. The proposed theory allows one to create a medium with a desirable spatially inhomogeneous permeability. The main new physical result is the explicit analytic formula for the permeability $\mu(x)$ of the limiting medium. The computational results confirm a possibility to create the media with various distributions of $\mu(x)$.
\end{abstract}

Index Terms-EM Wave Scattering, Desired Permeability, Modeling Results.

\section{INTRODUCTION}

The theory of electromagnetic (EM) wave scattering by many small impedance particles embedded in a homogeneous medium with a constant permittivity $\varepsilon_{0}>0$, permeability $\mu_{0}>0$ is applied for creating a media with a prescribed permeability. The computational procedures for numerical solution of the scattering problem were developed in [1], [2].

The small particles are embedded in a finite domain $D$. The medium, created by the embedding of the small particles, has new physical properties. In particular, it has a spatially inhomogeneous magnetic permeability $\mu(x)$, which can be controlled by the choice of the boundary impedances of the embedded small particles and their distribution density. An analytic formula for the permeability of the new medium is obtained.

We assume that in any sub-domain $\Delta$ of $D$, the number $N(\Delta)$ of the embedded particles is given by the formula:

$$
\mathcal{N}(\Delta)=\frac{1}{a^{2-\kappa}} \int_{\Delta} N(x) d x[1+o(1)], \quad a \rightarrow 0
$$

where $N(x) \geq 0$ is a continuous function, vanishing outside of the finite domain $D$ in which small particles are distributed, $\kappa \in(0,1)$ is a number that one can choose as one wishes, and the boundary impedances of the small particles are defined by the formula:

\author{
Mykhaylo Andriychuk \\ Pidstryhach Institute for Applied \\ Problems in Mechanics and Mathematics \\ 3"B” Naukova St., Lviv, 79060, Ukraine \\ E-mail: andr@iapmm.lviv.ua
}

$$
\zeta_{m}=\frac{h\left(x_{m}\right)}{a^{\kappa}}, \quad x_{m} \in D_{m}, \quad \operatorname{Re} h(x) \geq 0
$$

where $x_{m}$ is a point inside $m$-th particle, and $h(x)$ is a continuous function vanishing outside $D$, which satisfies only the physical restriction $\operatorname{Re} h(x) \geq 0$. The functions $N(x)$ and $h(x)$ can be chosen by the experimentalist. The function $h$, used in our numerical examples satisfies this restriction. The impedance boundary condition on the surface $S_{m}$ of the $m$ th particle $D_{m}$ is $E^{t}=\zeta_{m}\left[N, H^{t}\right]$, where $E^{t}\left(H^{t}\right)$ is the tangential component of $E(H)$ on $S_{m}$, and $N$ is the unit normal to $S_{m}$, pointing out of $D_{m}$.

Since one can choose the functions $N(x)$ and $h(x)$, one can create a desired magnetic permeability in $D$. This is a novel idea proposed originally in [6], [7]. It has led to a recipe for creating materials with a desired refraction coefficient in [9], [11].

Materials with negative permittivity and permeability are of interest in applications, see [4].

An equation for the effective EM field in the limiting medium is derived. This medium is created when the size $a$ of small particles tends to zero while the total number $M=M(a)$ of the particles tends to infinity at a suitable rate.

The proposed theory may be viewed as a "homogenization theory", but it differs from the usual homogenization theory (see, e.g., [3], and references therein) in several respects: we do not assume any periodic structure in the distribution of small bodies, our problem does not have a discrete spectrum, our operators are non-selfadjoint, to mention some of the differences. The ideas, methods, and techniques are also quite different from the usual methods. These ideas are similar to the ideas developed in papers [7], [8], [10], [11], [12].

However, the scattering of EM waves brought new technical difficulties which come from the vectorial nature of the boundary conditions. Our approach is valid for small particles of arbitrary shape, but for simplicity we assume that the small bodies are balls of radius $a$. 


\section{EM WAVE ScAtTering By MANy SMAll PARTiCles}

\section{A. Statement of Problem}

It is assumed that many small bodies $D_{m}, 1 \leq m \leq M$, are embedded in a homogeneous medium with constant parameters $\varepsilon_{0}, \mu_{0}$. Let $k^{2}=\omega^{2} \varepsilon_{0} \mu_{0}$, where $\omega$ is the frequency. Denote by $[E, H]=E \times H$ the cross product of two vectors, and by $(E, H)=E \cdot H$ the dot product of two vectors.

EM wave scattering problem consists of finding vectors $E$ and $H$ satisfying the Maxwell equations:

$$
\nabla \times E=i \omega \mu_{0} H, \quad \nabla \times H=-i \omega \varepsilon_{0} E
$$

in $D:=\mathrm{R}^{3} \backslash \bigcup_{m=1}^{M} \mathrm{D}_{m}$, the impedance boundary conditions:

$$
[N,[E, N]]=\zeta_{m}[N, H]
$$

on $S_{m}, 1 \leq m \leq M$, and the radiation conditions:

$$
E=E_{0}+v_{E}, \quad H=H_{0}+v_{H},
$$

where $\zeta_{m}$ is the impedance, $N$ is the unit normal to $S_{m}$ pointing out of $D_{m}, E_{0}, H_{0}$ are the incident fields satisfying equations (3) in all of $\mathrm{R}^{3}, S:=\cup_{m=1}^{M} S_{m}$.

One often assumes that the incident wave is a plane wave [5], i.e., $E_{0}=\mathcal{E} e^{i k \alpha \cdot x}, \mathcal{E}$ is a constant vector, $\alpha \in S^{2}$ is a unit vector, $S^{2}$ is the unit sphere in $\mathrm{R}^{3}, \alpha \cdot \mathcal{E}=0, v_{E}$ and $v_{H}$ satisfy the radiation condition:

$$
r\left(\frac{\partial v}{\partial r}-i k v\right)=o(1)
$$

Impedance $\zeta_{m}$ is assumed to be a constant, $\operatorname{Re} \zeta_{m} \geq 0$, so that

$$
\operatorname{Re}\left(\zeta_{m} E^{t}, E^{t}\right) \geq 0 \forall E^{t} \in T_{m},
$$

where $T_{m}$ is the set of all tangential to $S_{m}$ continuous vector fields, and $E^{t}$ is the tangential to $S$ component of $E$. Smallness of $D_{m}$ means that $k a \ll 1$, where

$$
a=0.5 \max _{1 \leq m \leq M} \operatorname{diam} D_{m} .
$$

Our definition of $E^{t}$ is:

$$
E^{t}=E-N(E, N)=[N,[E, N]] .
$$

This definition differs from the one used often in the literature. Since

$$
H=\frac{\nabla \times E}{i \omega \mu_{0}}
$$

one gets

$$
\nabla \times \nabla \times E=k^{2} E \quad \text { in } D,
$$

and the impedance boundary condition is

$$
[N,[E, N]]=\frac{\zeta_{m}}{i \omega \mu_{0}}[N, \nabla \times E]
$$

on $S_{m}, 1 \leq m \leq M$.

Thus, we have reduced problem (3)-(5) to finding one vector $E(x)$ satisfying the impedance boundary condition (11). If $E(x)$ is found, then $H=\frac{\nabla E}{i \omega \mu_{0}}$.

\section{B. Finding the Solution}

Let us look for $E$ of the form

$$
\begin{aligned}
& E=E_{0}+\sum_{m=1}^{M} \nabla \times \int_{S_{m}} g(x, t) J_{m}(t) d t, \\
& g(x, y)=\frac{e^{i k|x-y|}}{4 \pi|x-y|},
\end{aligned}
$$

where $t \in S_{m}$ and $d t$ is an element of the area of $S_{m}, J_{m}(t) \in$ $T_{m}$. This $E$ for any continuous $J_{m}(t)$ solves equation (10) in $D$ because $E_{0}$ solves (10).

Define the effective field $E_{e}(x)=E_{e}^{m}(x)=E_{e}^{(m)}(x, a)$, acting on the $m$-th body $D_{m}$, by the formula:

$$
E_{e}(x)=E(x)-\nabla \times \int_{S_{m}} g(x, t) J_{m}(t) d t:=E_{e}^{(m)}(x),
$$

where it is assumed that $x$ is in a neighborhood of $S_{m}$, but $E_{e}(x)$ is defined for all $x \in \mathrm{R}^{3}$. Let $x_{m} \in D_{m}$ be a point inside $D_{m}$, and $d=d(a)$ be the distance between two neighboring small bodies. Let us assume that

$$
\lim _{a \rightarrow 0} \frac{a}{d(a)}=0, \quad \lim _{a \rightarrow 0} d(a)=0 .
$$

It is proved in [7] that $E_{e}(x, a)$ tends to a limit $E_{e}(x)$ as $a \rightarrow$ 0 , and $E_{e}(x)$ is a twice continuously differentiable function.

Let us assume that in any sub-domain $\Delta$, the number $\mathcal{N}(\Delta)$ of the embedded bodies $D_{m}$ is given by formula (1), and boundary impedances $\zeta_{m}$ of small particles are defined by formula (2).

Let us write (12) as

$$
\begin{aligned}
& E(x)=E_{0}(x)+\sum_{m=1}^{M}\left[\nabla_{x} g\left(x, x_{m}\right), Q_{m}\right]+ \\
& \sum_{m=1}^{M} \nabla \times \int_{S_{m}}\left(g(x, t)-g\left(x, x_{m}\right)\right) J_{m}(t) d t,
\end{aligned}
$$

where

$$
Q_{m}:=\int_{S_{m}} J_{m}(t) d t
$$

One has $J_{m}=O\left(a^{-\kappa}\right)$ and $Q_{m}=O\left(a^{2-\kappa}\right)$. We prove that the second sum in (15) is negligible compared with the first one. This proof is based on several estimates. These estimates show that one may neglect the second sum in (15), and write

$$
E_{e}(x) \sim E_{0}(x)+\sum_{m=1}^{M}\left[\nabla_{x} g\left(x, x_{m}\right), Q_{m}\right], \quad a \rightarrow 0,
$$

with an error that tends to zero as $a \rightarrow 0$. When $\left|x-x_{j}\right| \sim a$ then the term with $m=j$ in the sum (17) should be dropped according to the definition of the effective field. 
As $a \rightarrow 0$, the sum in (17) converges to the integral

$$
E(x)=E_{0}(x)+\nabla \times \int_{D} g(x, y) N(y) Q(y) d y,
$$

where $Q(y)$ is the function uniquely defined by the formula

$$
Q_{m}=Q\left(x_{m}\right) a^{2-\kappa},
$$

and $Q(y)$ is a continuous function in $D$. The function $Q(y)$ is defined uniquely, because, as $a \rightarrow 0$ the set of points $\left\{x_{m}\right\}_{m=1}^{M}$ becomes dense in $D$. The physical meaning of vector $E(x)$ in equation (18) is clear: this vector is the limit of the effective field $E_{e}(x)$ as $a \rightarrow 0$, and $N(x)$ is the function from equation (1).

The function $Q(y)$ can be expressed in terms of $E$ :

$$
Q(y)=\frac{8 \pi i}{3 \omega \mu_{0}} h(y)(\nabla \times E)(y) .
$$

The factor $\frac{8 \pi}{3}$ appears if $D_{m}$ are balls. Otherwise a tensorial factor $c_{m}$, depending on the shape of $S_{m}$, should be used in place of $\frac{8 \pi}{3}$.

From equations (18) and (20) one obtains

$$
E(x)=E_{0}(x)+\frac{8 \pi i}{3 \omega \mu_{0}} \nabla \times \int_{D} g(x, y) h(y) N(y) \nabla \times E(y) d y
$$

\section{Explicit Formula for Permeability of Resulting Medium}

Let us derive the explicit formula for permeability of new medium using equation (21). Applying the operator $\nabla \times \nabla \times$ to (21), using the formula $\nabla \times \operatorname{grad}=0$ and the equation

$$
-\nabla^{2} g(x, y)=k^{2} g(x, y)+\delta(x-y) \text {, }
$$

and took into account that $h(x)$ is a scalar function by the assumption, we obtain

$$
\begin{aligned}
& \nabla \times \nabla \times E=K^{2}(x) E+\frac{\frac{8 \pi i}{3 \omega \mu_{0}}}{1-\frac{8 \pi i}{3 \omega \mu_{0}} h(x) N(x)} . \\
& {[\nabla(h(x) N(x)), \nabla \times E(x)],}
\end{aligned}
$$

where

$$
K^{2}(x)=\frac{k^{2}}{1-\frac{8 \pi i}{3 \omega \mu_{0}} h(x) N(x)}, \quad k^{2}=\omega^{2} \varepsilon_{0} \mu_{0} .
$$

If one uses equations with variable $\mu(x)$ :

$$
\nabla \times E=i \omega \mu(x) H, \quad \nabla \times H=-i \omega \varepsilon(x) E,
$$

then

$$
\nabla \times \nabla \times E=\omega^{2} \varepsilon(x) \mu(x) E+\left[\frac{\nabla \mu(x)}{\mu(x)}, \nabla \times E\right] .
$$

Comparing this equation with (23), one can identify the last term in (23) as coming from a variable permeability $\mu(x)$. This $\mu(x)$ appears in the limiting medium due to the boundary currents on the surfaces $S_{m}, 1 \leq m \leq M$. These currents appear because of the impedance boundary conditions (11).
Let us identify the permeability $\mu(x)$. Denote

$$
\Psi(x):=1-\frac{8 \pi i}{3 \omega \mu_{0}} h(x) N(x) .
$$

Let $\varepsilon(x)=\varepsilon_{0}, \varepsilon_{0}=$ const, and define

$$
\mu(x):=\frac{\mu_{0}}{\Psi(x)} .
$$

Then $K^{2}=\omega^{2} \varepsilon_{0} \mu(x)$, and

$$
\frac{\nabla \mu(x)}{\mu(x)}=\frac{\nabla \Psi(x)}{\Psi(x)} .
$$

Consequently, formula (23) has a clear physical meaning: the electromagnetic properties of the limiting medium are described by the variable permeability:

$$
\mu(x)=\frac{\mu_{0}}{\Psi(x)}=\frac{\mu_{0}}{1-\frac{8 \pi i}{3 \omega \mu_{0}} h(x) N(x)},
$$

and the limiting medium is described by the new refraction coefficient $K^{2}=\omega^{2} \varepsilon_{0} \mu(x)$.

\section{NUMERICAL RESUltS}

The proposed approach allows one to create a media with a piecewise-constant magnetic permeability $\mu$. Such permeability can be realized either by embedding various numbers $M_{m}$ of particles into sub-domains $\Delta_{m}$ or by the variation of the function $h\left(x_{m}\right)$ in these sub-domains.

Both approaches have their advantages depending on the parameters $M, a$, and $d$ of $D$. In engineering it is often useful to have constant distribution of $\mu$ along certain direction (for example, along $z-$ and $y$-axes), and piecewise-constant $\mu$ in the direction of $x$-axis.

In Fig. 1, such $\mu$ is formed by embedding various numbers of particles $M_{m}$ in sub-domains $\Delta_{m},(m=1,2,3): M_{1}=$ $11 \times 11 \times 4, M_{2}=9 \times 9 \times 3, M_{3}=11 \times 11 \times 4$, respectively. At these values of $M_{m}$, the values of permeability are equal to 0.9229 in sub-domains $\Delta_{1}$ and $\Delta_{3}$, and the permeability is equal to 0.9410 in sub-domain $\Delta_{2}$. Such distribution of particles in the medium allows one to reach a difference in the $\mu$ values in the range of $2.2 \%$, the minimal deviation of $\mu$ from the $\mu_{0}$ is $5.9 \%$. In order to increase the difference $\mu_{m}$ for various $D_{m}$ it is necessary to increase the difference between $M_{m}$. The normalized values of $\mu$ are shown here and in Fig. 2 and Fig. 3 below.

Combining the ratio of values $M_{m}$, one can create the distribution of $\mu$ corresponding to various requirements. For example, distribution of $\mu$ which is shown in Fig. 2, is obtained at the following values of particles in sub-domains $\Delta_{m}, m=1,2,3: M_{1}=11 \times 11 \times 4, M_{2}=9 \times 9 \times 3$, $M_{3}=7 \times 7 \times 4$. At such values of $M_{m}$, the values of the permeability are equal to $0.8745,0.9026,0.9451$ in subdomains $\Delta_{1}, \Delta_{2}$ and $\Delta_{3}$, respectively. The amplitudes $E_{x}$ and $E_{y}$ of EM field for this case have more complicated structure because of the larger change of $\mu$.

In Fig. 3, the piecewise-constant distribution of $\mu$, created by prescribing the various values of function $h\left(x_{m}\right)$ in four 


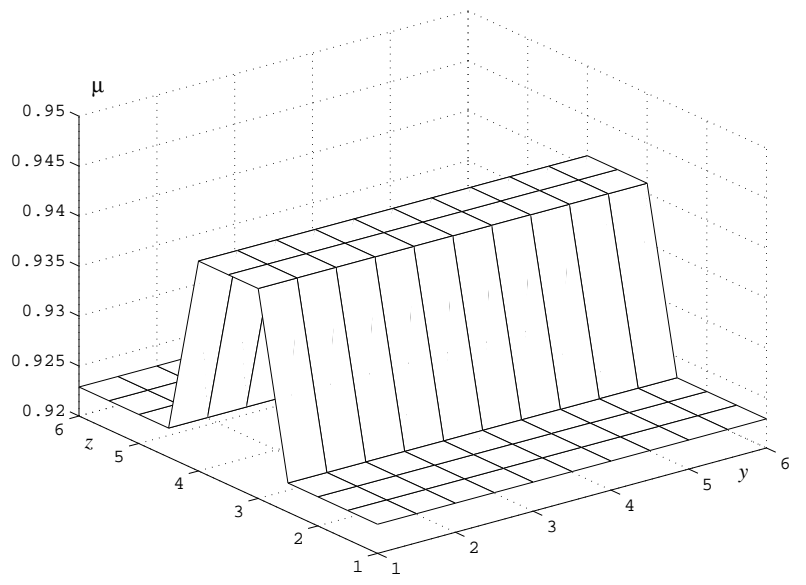

Fig. 1. The piecewise-constant distribution of $\mu$ corresponding to two various $M_{m}$

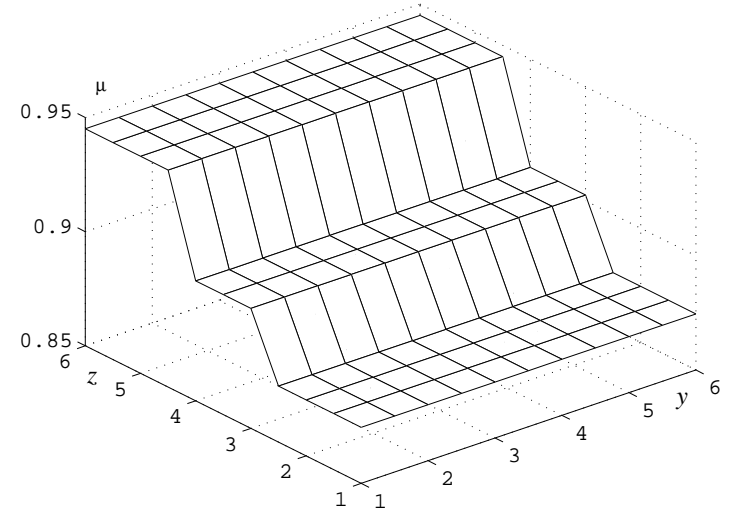

Fig. 2. The piecewise-constant distribution of $\mu$ corresponding to three various $M_{m}$

sub-domains $\Delta_{m},(m=1,2,3,4)$ of $D$, is shown. The number of particles in all sub-domains is equal to: $M=11 \times 5 \times 5$, the values of $h\left(x_{m}\right)$ are the following $h_{1}=7 i, h_{2}=9 i, h_{3}=11 i$, $h_{4}=13 i$ (that is, $h\left(x_{m}\right)$ is piecewise-constant). The minimal deviation of the obtained $\mu$ from the values $\mu_{0}$ is observed in sub-domain $\Delta_{1}$ and it is equal to $4 \%$, this deviation reaches $7 \%$ in sub-domain $\Delta_{4}$. In order to increase the deviation of $\mu$ in certain sub-domain $\Delta_{m}$ it is necessary to increase the value of $h_{m}$ in this sub-domain and keep the values of $h_{m}$ in the rest of sub-domains the same.

\section{CONCLUSIONS}

The numerical procedures for solving the EM wave scattering problem by many small impedance particles of an arbitrary shape are given. On this basis a method for creating the media with non-uniform distribution of magnetic permeability $\mu(x)$ is developed and tested numerically.

The computational results confirm the theoretical conclusion

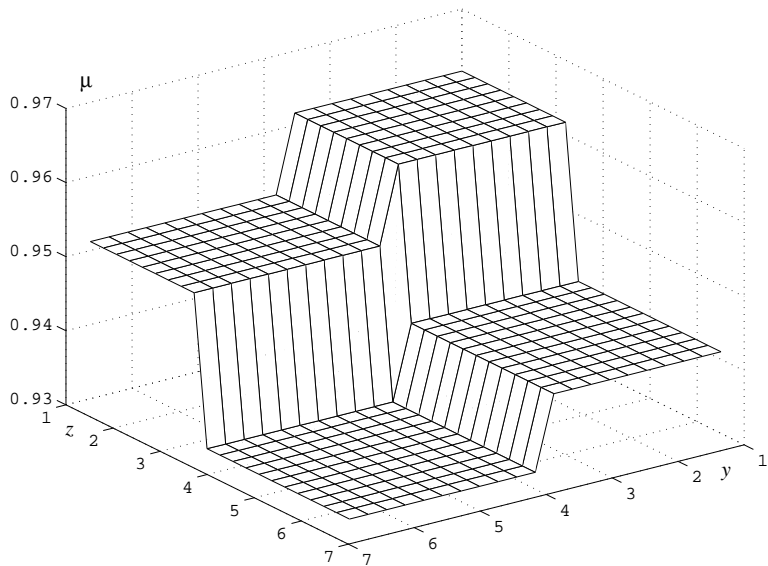

Fig. 3. The piecewise-constant distribution of $\mu$ corresponding to four various values of $h\left(x_{m}\right)$

about the possibility to create media with piecewise-constant permeability $\mu$. Creating such a $\mu$ is achieved either by embedding various numbers $M_{m}$ of small particles in the subdomains $\Delta_{m}$ of total domain $D$, or by changing the function $h\left(x_{m}\right)$ which determines the value of boundary impedances of the particles. Both approaches provide the possibility to change the initial values of $\mu$ in the range of $5 \%-40 \%$.

\section{REFERENCES}

[1] M. I. Andriychuk, A. G. Ramm, Scattering by Many Small Particles and Creating Materials with a Desired Refraction Coefficient. Int. J. Comput. Science and Math. 3, N 1/2, 102-121 (2010).

[2] M. I. Andriychuk, S. W. Indratno, A. G. Ramm, Electromagnetic Wave Scattering by a Small Impedance Particle: Theory and Modeling. Optics Communications. 285, 1684-1691 (2012).

[3] D. Cioranescu, P. Donate, An Introduction to Homogenization. Oxford Univ. Press, New York (1999).

[4] R. Marqués, J. Martel, F. Mesa, F. Medina, Left-Handed-Media Simulation and Transmission of EM Waves in Subwavelength SplitRing-Resonator-Loaded Metallic Waveguides. Phys. Rev. Lett. 89(18), 183901-1-183901-4 (2002).

[5] A. G. Ramm, Scattering by Obstacles. D. Reidel, Dordrecht (1986).

[6] A. G. Ramm, Distribution of Particles which Produces a "Smart" Material. J. Stat. Phys. 127, N 5, 915-934 (2007).

[7] A. G. Ramm, Many-body wave scattering by small bodies and applications. J. Math. Phys. 48, N 10 (2007).

[8] A. G. Ramm, Wave Scattering by Many Small Particles Embedded in a Medium. Phys. Lett. A. 372(17), 3064-3070 (2008).

[9] A. G. Ramm, Many-body Wave Scattering Problems in the Case of Small Scatterers. J. of Appl. Math. and Comput. 41, N 1, 473-500 (2013).

[10] A. G. Ramm, Electromagnetic Wave Scattering by a Small Impedance Particle of Arbitrary Shape. Optics Communications. 284, 3872-3877 (2011).

[11] A. G. Ramm, Scattering of Acoustic and Electromagnetic Waves by Small Bodies of Arbitrary Shapes. Applications to Creating New Engineered Materials, Momentum Press, New York, 2013.

[12] A. G. Ramm, Electromagnetic wave scattering by small impedance particles of an arbitrary shape and applications. Challenges. 5, 35-42 (2014) 